\title{
On the use of URLs and hashtags in age prediction of Twitter users
}

\author{
Abhinay Pandya, Mourad Oussalah \\ Center for Ubiquitous Computing \\ University of Oulu, Finland \\ abhinay.pandya@oulu.fi \\ mourad.oussalah@oulu.fi
}

\author{
Paola Monachesi \\ University of Utrecht, Netherlands \\ p.monachesi@uu.nl
}

\author{
Panos Kostakos, Lauri Lovén \\ Center for Ubiquitous Computing \\ University of Oulu, Finland \\ panos.kostakos@oulu.fi \\ luari.loven@oulu.fi
}

\begin{abstract}
Social media data represent an important resource for behavioral analysis of the ageing population. This paper addresses the problem of age prediction from Twitter dataset, where the prediction issue is viewed as a classification task. For this purpose, an innovative model based on Convolutional Neural Network is devised. To this end, we rely on languagerelated features and social media specific metadata. More specifically, we introduce two features that have not been previously considered in the literature: the content of URLs and hashtags appearing in tweets. We also employ distributed representations of words and phrases present in tweets, hashtags and URLs, pre-trained on appropriate corpora in order to exploit their semantic information in age prediction. We show that our CNN-based classifier, when compared with an SVM baseline model, yields an improvement of $12.3 \%$ and $6.6 \%$ in the micro-averaged F1 score on the Dutch and English datasets, respectively.
\end{abstract}

Keywords-Social Media Mining, Twitter, Convolutional Neural Networks, Age prediction

\section{INTRODUCTION}

Social media data represent an important resource for behavioral analysis, especially in the case of the ageing population since it allows us to understand its needs and interests and to support healthy ageing. Indeed, identifying appropriate age group enables authorities to design relevant course of action as the interests and needs trivially vary with age. More specifically, in the context of the Grage project (www.grageproject.eu), we are interested in identifying different age groups since issues concerning healthy lifestyle and urban life might be faced differently from different communities (e.g., age groups).

However, finding biographical information of users is difficult. Many social media platforms hide it behind complex access control policies, making necessary to develop sophisticated algorithms that can infer biographical attributes, such as age, from text, which is a challenging task. First, because age is a social and fluid variable[2] that is shaped depending on the societal context, the culture of the speakers, the individual experiences, and the multitude of social roles[3].

Language can be used as a resource to construct people's identity making it an important means to detect behavioral and biographical information due to the availability of public data through social media platforms such as Twitter.
Previous work ([4],[3],[5],[6],[7],[8],[9],[10]) has identified several features based on both language and social media specific metadata that are relevant for the age prediction task including bag-of-words, linguistic features, stylometric features, profile features, (e.g., background color, profile image), social network, and preferences (e.g., liked tweets).

In this paper, we focus on Twitter data and we build on previous work on age prediction by relying on language related features and social media metadata to classify users in age groups, considering thus age as a categorical variable. Age prediction can be viewed as a regression (for exact numeric age prediction) or classification (for age-category prediction) task. Since in the context of the Grage project we are interested in age-groups rather than exact age values, we model the problem as a classification task. Many approaches based on such multi-class classification have been proposed, such as SVMs ([11],[12]), logistic regression ([4],[13]) and Naive Bayes ([14]). Nevertheless as pointed out by [3], the problem of age estimation is very challenging due to inherent variability of human language together with unstructured text in tweet messages, which raises the question of finding appropriate cues that elicit categorical age and the subsequent reasoning. This motivates the current work in this paper. Especially, the following contributions are highlighted:

1) CNN-based model for classification: we propose an innovative model based on convolutional neural networks $(\mathrm{CNN}))[15]$ that integrates heterogenous features for age-category classification.

2) Hashtags and URLs, as new features: to the best of our knowledge, there is no previous work using the content of hashtags and URLs for age prediction. Although they have been considered as social media specific features (i.e. their number has been included in [4]), their content has not been considered. We contend that hashtags and URLs in tweets are indicative of users' age since they reflect users' interests and activities[1]. We propose a novel method to derive relevant features from hashtags and URLs and incorporate these into our CNN-based classification model.

3) Enhanced semantics through pre-trained word em- 
beddings: Unsupervised learning of distributed representations (word embeddings) obviates the need for careful feature engineering and such representations are richer in semantic information than standard bagof-words [16],[17],[18]. We propose to employ word embeddings for tweet texts, title text of the pages referred to by the URLs in the tweets, and for most frequently co-occurring words with each hashtag in the tweet. Furthermore, we pre-train word embeddings on different corpora to take the context into account. More specifically, word embedding vectors used for tweet texts and hashtags are pre-trained on large collection of tweets, and those used for URLs are pre-trained on blogs/news.

We employ two existing datasets from two different languages (i.e. English and Dutch) to test the validity of our novel features and classification method against the versatile classification model SVM as a baseline and show that our CNN-based model outperforms the baseline significantly. The rest of the paper is organized as follows. In Section 2, we discuss related work. Section 3 depicts our approach to extract features for age prediction of Twitter users and describes our CNN-based model. Section 4 explains our experimental setup and results compared with baseline approaches. Finally, in Section 5, we present our conclusions.

\section{RELATED WORK}

Linguistic literature such as [19] has played an important role in identifying areas of change in language use that could be formalized as features to predict age. Examples include emotional processes, social and identity relationships, time orientation, and cognitive complexity. Similarly, [20] focuses on linguistic features such as slang and words indicating personal feelings. Slang is employed the most by teen users ([21], [22], [13]) similarly for chat words (i.e. abbreviations, acronyms). Literature suggests that chat language follows the same trend as slang and is used most by younger users, with teens using it the most ([23], [11], [4]). [5] employ an open vocabulary approach to detect age, that is they do not rely on an already predefined set of words that correlate with age classes. They find language features through a differential language analysis that makes it possible to extract words, phrases (i.e. n-grams of size 1 to 3 ) and topics derived using LDA, directly from the text.

Social media also exhibit medium-specific features (i.e. metadata) that show a different use wrt. age, as is the case for sharing links and/or images and tagging/hashtag use. Authors in [24] have studied blog posts and showed that there is no trend in image sharing, but there is a gentle increase in usage of URLs in posts with respect to age: apart from an inexplicable peak at the age of 24 , link sharing increases with age with users older than 35 posting the most. This result on URLs is supported also by [23],[19], who have continued research on blogging and found that the sharing of links increases with age. On the other hand, work in [4] demonstrated that a sharp rise in the use of links for Dutch Twitter users in their 20s, that stagnates in their 30s. They associated this finding with information sharing and impression management. Rosenthal and McKeown[13] showed that the use of links and images in their blog data varies across all ages. In the case of hashtags, Nguyen et al.[4] found that hashtags are used more often by older Twitter users: low usage in teens, a steep climb in the 20 s, the highest and continuous use through the years up until the oldest participants category (over 60 years of age). According to these authors, hashtags are, similarly to links, connected to the sharing of information and older tweeters apparently are more concerned with information sharing than younger users. Younger people seem to display a certain kind of online identity, something older people are less concerned with[25].

Computational work on age prediction has exploited these differences in the use of URL's and hashtags across age groups, but have not considered the content associated to them, that also reflects an age related use. For example, [26] have researched the behaviour of two groups on Instagram: the adult group (25-39) displays a wider range of interests in topics and are very diverse: arts/photos/design, locations, mood/emotion, nature, social/people. The majority of the teens' (13-19) hashtags concern mood/emotion and follow/like. Monachesi and Leeuw[1] concluded that hashtags are an important feature to discriminate age since older adults above 67 use mainly hashtags related to politics and leisure in Twitter while people below 55, use mainly hashtags in the context of work related activities and technology.

\section{APPROACH}

\section{A. Feature extraction method}

Our approach, to include the content of URLs and hashtags as novel features, in combination with word embeddings is summarized in Algorithms 1, 2 and 3. We hypothesize that it can play a role in the age detection task from Twitter data since it includes semantic information that reflects the interests of Twitter users that change with age. More specifically:

1) We extract the 200 most recent tweets for each user to ensure a large enough dataset. We name this set the TweetTextSet.

2) We expand all URLs and hashtags in the TweetTextSet as follows:

- We fetch the title of each linked web page. We name this set of titles the URLTitlesSet. We use a metadata_parser ${ }^{1}$ for fetching the titles of the webpages pointed to by the URLs in tweets.

\footnotetext{
${ }^{1}$ https://github.com/jvanasco/metadata_parser
} 
- For each hashtag, we collect $N$ tweets containing this hashtag. Since hashtags have different meanings at different times, we use a time window of $+/-10$ days, counting from the date of the tweet mentioning the hashtag. This set of $N$ tweets then defines the semantic context of that hashtag. We then perform co-occurrence analysis to find and select the most frequently co-occurring words/phrases with each hashtag. We name this set of $M$ words/phrases as HashtagCooccurringSet. HashtagCooccurringSet serves as a representative set of topics the user is interested in since it covers all hashtags cited in her recent 200 tweets.

3) We capture distributional semantics with word embeddings of words/phrases as follows:

- We pre-train a word2vec model on a large collection of tweets and another word2vec model on GoogleNews/blogs/forums.

- For each of the three sets TweetTextSet, URLTitlesSet, and HashtagCooccurringSet, we look up in pre-trained dictionaries to replace occurrences of words and phrases with their corresponding vectors. We ignore unknown words and phrases. For TweetTextSet and HashtagCooccurringSet, we use word2vec model trained on tweets, and for URLTitlesSet, we use the model trained on GoogleNews/blogs/forums.

We use two different word2vec dictionaries to capture the semantics of the relevant contexts: for words and phrases in tweet texts, the model trained on tweets is a better fit than a model trained on generic texts such as blogs, news or forums. More specifically, in order to map Dutch TweetTextSet to the corresponding word embedding vectors, we pre-trained a word2vec model (200 dimensions) from 4.3 million Dutch tweets using Gensim library. ${ }^{2}$ To map Dutch URLTitlesSet to the corresponding word embedding vectors, we utilize a pre-trained model from LREC2016 (320 dimensions). ${ }^{3}$ These vectors were trained on Wikipedia, COW, Sonar500, and other corpora. To map English TweetTextSet to the corresponding word embedding vectors, we use a Twitter word2vec model trained on 400 million tweets. ${ }^{4}$ For mapping English URLTitlesSet to the corresponding word embedding vectors, we use pre-trained word2vec vectors (300 dimensions) on GoogleNews. ${ }^{5}$

4) We calculate the min, $\max$, and avg vectors of the hashtag word embeddings (HashtagCooccurringSet): $\min$ is the minimum value across all hashtag word

\footnotetext{
${ }^{2} \mathrm{https}: / /$ radimrehurek.com/gensim/index.html

${ }^{3}$ https://github.com/clips/dutchembeddings

${ }^{4}$ https://github.com/loretoparisi/word2vec-twitter

${ }^{5}$ https://code.google.com/archive/p/word2vec/
}

embedding vectors in each dimension of the vector, max is the maximum value, and avg is the average value. Avoiding computationally expensive option to find basis vectors for the span of word embedding vectors of HashtagCooccurringSet, we choose these three vectors as capturing the semantic space associated with user's hashtags which represents user's interests and activities.

5) We fetch Twitter metadata (e.g. tweet timestamps, see Section III-B for more details) for each user.

6) We extract additional features such as linguistic, stylometric, and Twitter-specific (see Section III-B for more details) from the metadata and TweetTextSet. We create a normalized vector of these additional features. For English tweets, we use the Carnegie Mellon's TweetNLP suite ${ }^{6}$ to tokenize the tweets and to assign Part-of-speech tags. For Dutch tweets, we use Frog ${ }^{7}$ for tokenization and POS tagging.

7) We incorporate the word embeddings from TweetTextSet and URLTitlesSet and the additional features vector, as described above, into a Convolutional Neural Network based model for classification (see Section III-C for further information).

\section{B. Additional language and social media features}

In addition to the content of URLs/hashtags and the distributed representations of words and phrases, we employ the following language and social media specific features already proposed in previous literature([4],[3],[5],[6],[7],[8],[9],[10]):

\section{- Language features:}

- Linguistic Features: part-of-speech (POS) n-grams.

- Stylometric Features: average sentence length, average word length, ratio of the number of emoticons to the number of words, number of elongated words (and non-standard spellings) used, ratio of the number of hashtags to the number of words, number of slang words, number of acronyms.

- Features from pre-trained lexica: Researchers in sociolinguistcs have derived lexicons of words and phrases that correlate with different age groups. We use two such resources: EMNLP2014[27], and WWBP[5]. We use logit transformed values from these lexicons.

\section{- Twitter-specific features:}

- Tweet Features: earliest, latest, and average timestamp from among all 200 tweets of a user, number of geo-locatable tweets of a user, number of tweets favorited, number of tweets which are in-reply-to, number of tweets which are re-tweets, number of user mentions.

\footnotetext{
${ }^{6} \mathrm{http} / / / \mathrm{www} . c s . c m u . e d u /$ ark/TweetNLP

${ }^{7} \mathrm{http}: / /$ languagemachines.github.io/frog/
} 

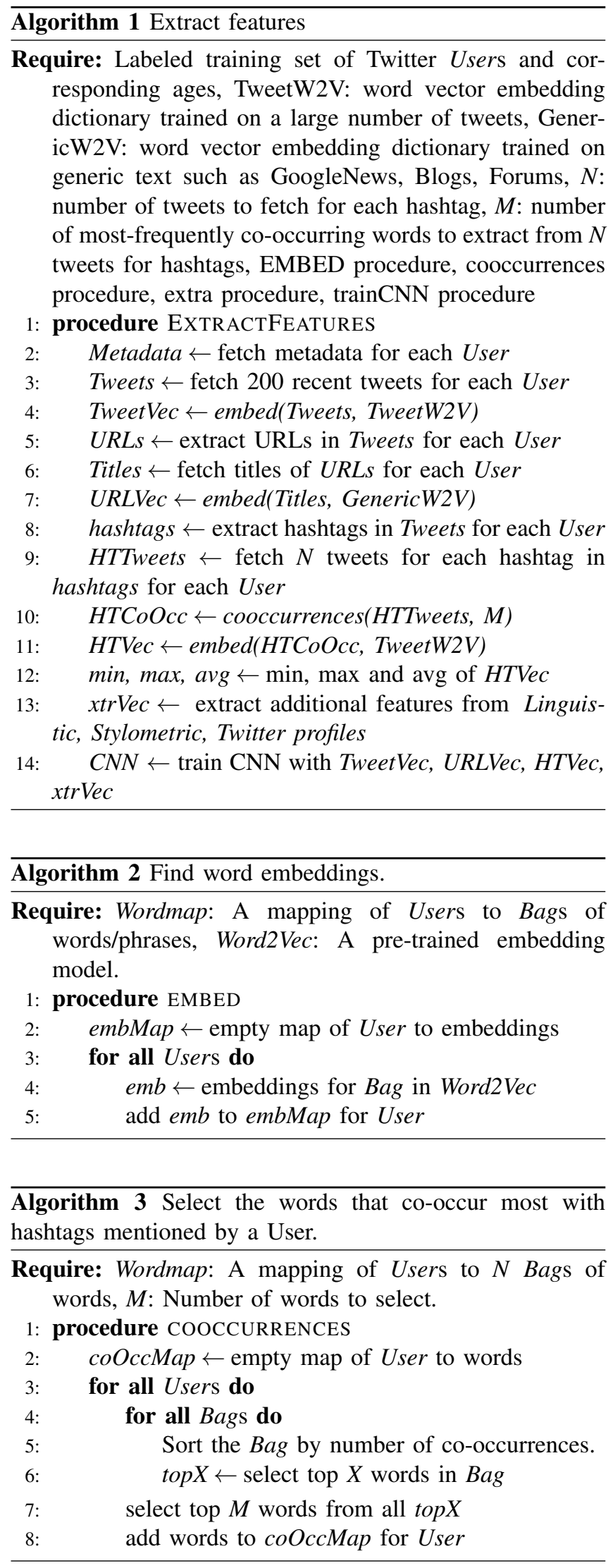

- Twitter user profile features: account creation date, listed-count, verified or not, geo-enabled or not, status-count.

- Twitter social network features: number of friends, number of followers, ratio of the number of friends to the number of followers, number of friends or followers with directed tweet exchanges, number of friends that are also followers.

\section{Convolutional Neural Network model}

Inspired by [15],[28] Figure 1 illustrates our model based on convolutional neural network $(\mathrm{CNN})$ for predicting the age category of a Twitter user. Our model is innovative in two aspects:

- Two separate input channels receive inputs from word embeddings of TweetTextsSet and URLTitlesSet and separate convolution filters of various sizes were used. This innovative design is proposed to prevent learning false associations between tweet words and words from the titles of webpages. The output of convolutional layer is passed through a non-linear activation function ReLU. Pooling layer aggregates vector elements by taking the maximum from each element of the convolutional feature map. Thus, these two output vectors after max-pooling represent features extracted from tweet texts and URLs for age-category prediction.

- Since CNNs require fixed-sized homogenous data sources, in order to utilize additional features, we propose annother design innovation: the two vectors described above are concatenated with (a) a vector representing the additional features (section III-B), (b) the three vectors min, max, and avg calculated from word embeddings of HashtagCooccurringSet, and (c) a vector of features from pre-created lexicons after normalizing values to logits $(-1$ to +1$)$. This concatenated vector is then fully connected with the output layer in soft-max setup. Since this vector is huge, we use dropout method for regularization.

\section{CNN Training details}

In order to train the above model, for each dataset, data was split into $85 \%$ training and $15 \%$ for validation sample. Batch size for training CNN was kept at 200 tweets. Since tweets differ in length, we limited each tweet to a minimum of 30 tokens. If a tweet was too short, we padded the tweet with a special PAD token. Since each tweet is different in content from others even by the same user, we carefully adjusted the sizes of kernel masks in order not to learn spurious features. In this way, we ensured that the convolution kernel masks did not move over different tweets. We limited URL title words to 25. If a URL title was shorter, we again padded the title with a special PAD token. If a title was longer than 25 words, we ignored the rest of the words. Since search engines typically display the first 50-60 


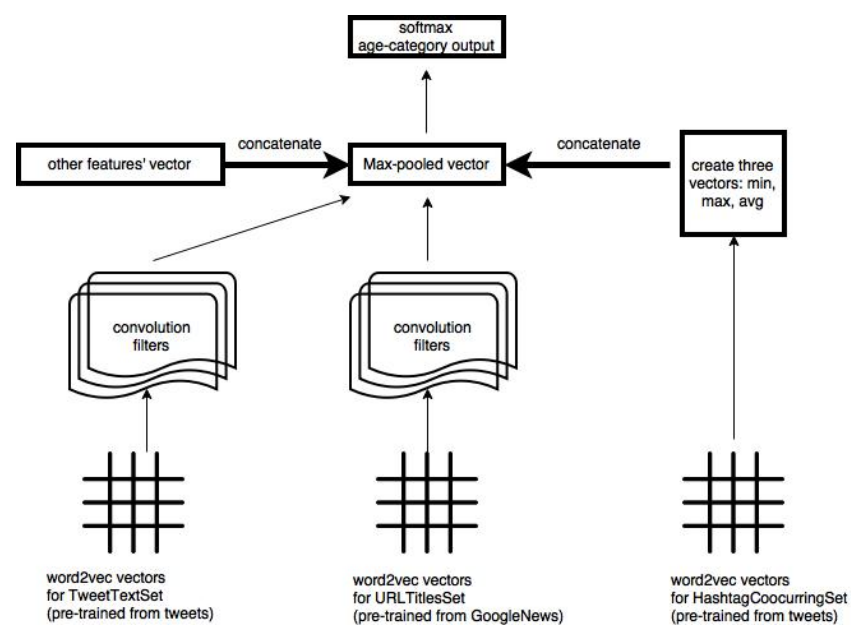

Figure 1: Our CNN Model for age-category classification.

characters of the titles, most web pages do not have titles exceeding the length of 25 words. Also, in our two datasets, we found that $93 \%$ titles are of shorter length than 25 words. The following choice of hyperparameters was made: for both datasets, we use $R e L U$ as non-linear activation function, filter windows of sizes 3, 4, 5, 6 with 128 feature maps each, and mini-batch size of 200. The loss is minimized using the Adam optimizer[29]. For regularization, we use the dropout method proposed by [30] after the max pooling layer with $p=0.5$. In addition, we complement this method of regularization with $L 2$-Regularization of softmax parameters.

\section{EXPERIMENTS AND RESULTS}

\section{A. Datasets}

In order to classify a user into an age group we need a dataset with (a) Twitter user id's, and (b) corresponding ages. A major problem for the age prediction task in Twitter is the limited availability of validated data annotated with the age of users. We use two datasets for Dutch and English partially derived from previous work of [4] and [6].

[4] sampled Dutch Twitter users in the fall of 2012. They employed external annotators to annotate the chronological age using information available through tweets, the Twitter profile and external social media profiles such as Facebook and LinkedIn. In total, over 3000 Twitter users were annotated. However, not all of these Twitter profiles are currently active, leaving us with the profile information of 1365 users that we have included in our Dutch dataset.

For the English corpus, similar to [6] and [7], we capture self-reported birthday tweets using search string "Happy nth Birthday", and we adopt the same age group categorization they have proposed.

More information about both datasets can be found in Table I. Because of differences in the datasets used in [4],[6] and our re-created datasets, a direct comparison of our results with theirs is not possible.
Table I: Dataset description

\begin{tabular}{|l|r|l|r|}
\hline \multicolumn{2}{|l|}{ Dutch dataset } & \multicolumn{2}{l|}{ English dataset } \\
\hline Age Group & No. of Users & Age Group & No. of Users \\
\hline $0-20$ & 786 & $13-17$ & 966 \\
\hline $20-40$ & 364 & $18-24$ & 1,324 \\
\hline 40 plus & 215 & 25 plus & 455 \\
\hline Total & 1,365 & Total & 2,745 \\
\hline
\end{tabular}

\section{B. Baselines}

In order to evaluate the performance of our CNN-based model with the new features proposed on the above datasets, we compare the results with those obtained from Support Vector Machines (SVM) classifier (with linear kernel) using various combinations of features. We also use the additional language and social media specific features that have been utilized in the previous works.

- B1: Lexical (Bag-of-words(BoW)) features with SVM: Unigrams, bigrams, and trigrams extracted from only tweet texts.

- B2: B1 and BoW representation of most frequently cooccurring words with hashtags in user's tweets (HashtagCooccurringSet)

- B3: B1 and BoW representation of words from titles of the URLs in user's tweets (URLTitlesSet)

- B4: B1 and Linguistic, Stylometric, Twitter-specific features

- B5: all features from B1, B2, B3, B4

- B6: B5 and features derived from external pre-trained age-specific lexica

\section{Results}

Tables II and III collect baseline results on English and Dutch datasets. Included are the precision, recall, and macro- and micro-averaged F1 scores obtained with SVM classification with various feature combinations. Because of the significant differences in the number of samples in each age-group, micro-averaged F1 score serves as a better indicator of classification accuracy.

Tables IV and V show results with our novel features integrated into our CNN-based model. In order to compare results of our new classification model and new representations of features with results obtained on baseline, we use the following feature combinations for our model:

- W1: word embeddings (pre-trained on tweets) of words/phrases from TweetTextSet

- W2: W1 to the input layer of CNN and min, max, and avg vectors derived from word embeddings of HashtagCooccurringSet concatenated with the maxpooled vector before soft-max

- W3: W1 to one branch of the input layer of CNN and word embeddings of URLTitlesSet to the other branch of input layer 
Table II: Baseline Results on Dutch dataset.

B1-B6 are explained in subsection IV-B

\begin{tabular}{|c|c|c|c|c|c|c|}
\hline & B1 & B2 & B3 & B4 & B5 & B6 \\
\hline Age $0-20$ & $\begin{array}{l}P: 0.80 \\
R: 0.81 \\
F: 0.805\end{array}$ & $\begin{array}{l}P: 0.78 \\
R: 0.83 \\
F: 0.804\end{array}$ & $\begin{array}{l}P: 0.77 \\
R: 0.82 \\
F: 0.794\end{array}$ & $\begin{array}{l}P: 0.81 \\
R: 0.84 \\
F: 0.82\end{array}$ & $\begin{array}{l}P: 0.82 \\
R: 0.84 \\
F: 0.83\end{array}$ & $\begin{array}{l}P: 0.83 \\
R: 0.85 \\
F: 0.84\end{array}$ \\
\hline Age $20-40$ & $\begin{array}{l}P: 0.58 \\
R: 0.64 \\
F: 0.608\end{array}$ & $\begin{array}{l}P: 0.52 \\
R: 0.69 \\
F: 0.593\end{array}$ & $\begin{array}{l}P: 0.51 \\
R: 0.63 \\
F: 0.563\end{array}$ & $\begin{array}{l}P: 0.60 \\
R: 0.66 \\
F: 0.63\end{array}$ & & $\begin{array}{l}P: 0.61 \\
R: 0.67 \\
F: 0.64\end{array}$ \\
\hline $\begin{array}{l}\text { Age } 40 \\
\text { plus }\end{array}$ & $\begin{array}{l}P: 0.84 \\
R: 0.36 \\
F: 0.50\end{array}$ & $\begin{array}{l}P: 0.78 \\
R: 0.40 \\
F: 0.53\end{array}$ & $\begin{array}{l}P: 0.80 \\
R: 0.41 \\
F: 0.54\end{array}$ & & & $\begin{array}{l}P: 0.87 \\
R: 0.45 \\
F: 0.59\end{array}$ \\
\hline $\begin{array}{l}\text { Macro- } \\
\text { averaged }\end{array}$ & $\begin{array}{l}P: 0.74 \\
R: 0.60 \\
F: 0.66\end{array}$ & $\begin{array}{l}P: 0.69 \\
R: 0.64 \\
F: 0.66\end{array}$ & $\begin{array}{l}P: 0.69 \\
R: 0.62 \\
F: 0.65\end{array}$ & $\begin{array}{l}P: 0.75 \\
R: 0.64 \\
F: 0.67\end{array}$ & $\begin{array}{l}P: 0.77 \\
R: 0.64 \\
F: 0.70\end{array}$ & $\begin{array}{l}P: 0.77 \\
R: 0.66 \\
F: 0.71\end{array}$ \\
\hline $\begin{array}{l}\text { Micro- } \\
\text { averaged }\end{array}$ & $\begin{array}{l}P: 0.76 \\
R: 0.65 \\
F: 0.70\end{array}$ & $\begin{array}{l}P: 0.73 \\
R: 0.67 \\
F: 0.70\end{array}$ & $\begin{array}{l}P: 0.72 \\
R: 0.68 \\
F: 0.70\end{array}$ & $\begin{array}{l}P: 0.76 \\
R: 0.67 \\
F: 0.71\end{array}$ & $\begin{array}{l}P: 0.76 \\
R: 0.69 \\
F: 0.72\end{array}$ & $\begin{array}{l}P: 0.77 \\
R: 0.69 \\
F: 0.73\end{array}$ \\
\hline
\end{tabular}

Table III: Baseline Results on English dataset.

B1-B6 are explained in subsection IV-B

\begin{tabular}{|c|c|c|c|c|c|c|}
\hline & B1 & B2 & B3 & B4 & B5 & B6 \\
\hline Age $13-17$ & $\begin{array}{l}P: 0.68 \\
R: 0.70 \\
F: 0.69\end{array}$ & $\begin{array}{l}P: 0.67 \\
R: 0.72 \\
F: 0.694\end{array}$ & $\begin{array}{l}P: 0.66 \\
R: 0.72 \\
F: 0.689\end{array}$ & $\begin{array}{l}P: 0.70 \\
R: 0.74 \\
F: 0.72\end{array}$ & $\begin{array}{l}P: 0.71 \\
R: 0.76 \\
F: 0.73\end{array}$ & $\begin{array}{l}P: 0.72 \\
R: 0.76 \\
F: 0.74\end{array}$ \\
\hline Age 18-24 & $\begin{array}{l}P: 0.77 \\
R: 0.73 \\
F: 0.75\end{array}$ & $\begin{array}{l}P: 0.75 \\
R: 0.76 \\
F: 0.755\end{array}$ & $\begin{array}{l}P: 0.74 \\
R: 0.75 \\
F: 0.745\end{array}$ & $\begin{array}{l}P: 0.79 \\
R: 0.77 \\
F: 0.78\end{array}$ & $\begin{array}{l}P: 0.80 \\
R: 0.80 \\
F: 0.80\end{array}$ & $\begin{array}{l}P: 0.81 \\
R: 0.80 \\
F: 0.81\end{array}$ \\
\hline $\begin{array}{l}\text { Age } 25 \\
\text { plus }\end{array}$ & $\begin{array}{l}P: 0.61 \\
R: 0.64 \\
F: 0.62\end{array}$ & $\begin{array}{l}P: 0.59 \\
R: 0.67 \\
F: 0.609\end{array}$ & $\begin{array}{l}P: 0.59 \\
R: 0.64 \\
F: 0.614\end{array}$ & $\begin{array}{l}P: 0.63 \\
R: 0.67 \\
F: 0.65\end{array}$ & $\begin{array}{l}P: 0.66 \\
R: 0.70 \\
F: 0.68\end{array}$ & $\begin{array}{l}P: 0.65 \\
R: 0.72 \\
F: 0.69\end{array}$ \\
\hline $\begin{array}{l}\text { Macro- } \\
\text { averaged }\end{array}$ & $\begin{array}{l}P: 0.69 \\
R: 0.69 \\
F: 0.69\end{array}$ & $\begin{array}{l}P: 0.67 \\
R: 0.72 \\
F: 0.69\end{array}$ & $\begin{array}{l}P: 0.66 \\
R: 0.70 \\
F: 0.68\end{array}$ & $\begin{array}{l}R: 0.71 \\
R: 0.73 \\
F: 0.72\end{array}$ & $\begin{array}{l}P: 0.72 \\
R: 0.75 \\
F: 0.73\end{array}$ & $\begin{array}{l}P: 0.73 \\
R: 0.76 \\
F: 0.74\end{array}$ \\
\hline $\begin{array}{c}\text { Micro- } \\
\text { averaged }\end{array}$ & $\begin{array}{l}P: 0.71 \\
R: 0.71 \\
F: 0.71\end{array}$ & $\begin{array}{l}P: 0.70 \\
R: 0.73 \\
F: 0.72\end{array}$ & $\begin{array}{l}P: 0.70 \\
R: 0.72 \\
F: 0.71\end{array}$ & $\begin{array}{l}P: 0.73 \\
R: 0.74 \\
F: 0.74\end{array}$ & $\begin{array}{l}P: 0.74 \\
R: 0.76 \\
F: 0.75\end{array}$ & $\begin{array}{l}P: 0.75 \\
R: 0.76 \\
F: 0.76\end{array}$ \\
\hline
\end{tabular}

- W4: W1 to the input layer of $\mathrm{CNN}$ and a vector constructed from Linguistic, Stylometric, and Twitterspecific features concatenated with the max-pooled vector before soft-max

- W5: word embeddings from TweetTextSet and URLTitlesSet as two branches of input layer, and all other features including min, max, and avg from HashtagCooccurringSet concatenated with the max-pooled vector before soft-max

- W6: same setup as W5 but with additional features derived from external pre-trained age-specific lexica concatenated with the max-pooled vectors
Table IV: Results on Dutch dataset with our CNN-based model with novel features.

W1-W6 are explained in the text

\begin{tabular}{|c|c|c|c|c|c|c|}
\hline & $W 1$ & $W 2$ & $W 3$ & $W 4$ & $W 5$ & $W 6$ \\
\hline Age 0-20 & $\mathrm{P}: 0.82$ & $\mathrm{P}: 0.84$ & $\mathrm{P}: 0.85$ & $\mathrm{P}: 0.83$ & $\mathrm{P}: 0.87$ & $\mathrm{P}: 0.88$ \\
& $\mathrm{R}: 0.87$ & $\mathrm{R}: 0.89$ & $\mathrm{R}: 0.91$ & $\mathrm{R}: 0.85$ & $\mathrm{R}: 0.91$ & $\mathrm{R}: 0.92$ \\
& $\mathrm{~F}: 0.84$ & $\mathrm{~F}: 0.86$ & $\mathrm{~F}: 0.88$ & $\mathrm{~F}: 0.84$ & $\mathrm{~F}: 0.89$ & $\mathrm{~F}: 0.90$ \\
\hline Age 20-40 & $\mathrm{P}: 0.60$ & $\mathrm{P}: 0.62$ & $\mathrm{P}: 0.63$ & $\mathrm{P}: 0.61$ & $\mathrm{P}: 0.66$ & $\mathrm{P}: 0.68$ \\
\hline & $\mathrm{R}: 0.68$ & $\mathrm{R}: 0.72$ & $\mathrm{R}: 0.73$ & $\mathrm{R}: 0.71$ & $\mathrm{R}: 0.74$ & $\mathrm{R}: 0.75$ \\
\hline & $\mathrm{F}: 0.64$ & $\mathrm{~F}: 0.68$ & $\mathrm{~F}: 0.69$ & $\mathrm{~F}: 0.67$ & $\mathrm{~F}: 0.71$ & $\mathrm{~F}: 0.71$ \\
\hline Age 40 & $\mathrm{P}: 0.83$ & $\mathrm{P}: 0.85$ & $\mathrm{P}: 0.87$ & $\mathrm{P}: 0.83$ & $\mathrm{P}: 0.89$ & $\mathrm{P}: 0.88$ \\
\hline plus & $\mathrm{R}: 0.45$ & $\mathrm{R}: 0.49$ & $\mathrm{R}: 0.52$ & $\mathrm{R}: 0.48$ & $\mathrm{R}: 0.57$ & $\mathrm{R}: 0.60$ \\
& $\mathrm{~F}: 0.58$ & $\mathrm{~F}: 0.62$ & $\mathrm{~F}: 0.63$ & $\mathrm{~F}: 0.61$ & $\mathrm{~F}: 0.69$ & $\mathrm{~F}: 0.71$ \\
\hline Macro- & $\mathrm{P}: 0.75$ & $\mathrm{P}: 0.77$ & $\mathrm{P}: 0.78$ & $\mathrm{P}: 0.76$ & $\mathrm{P}: 0.80$ & $\mathrm{P}: 0.81$ \\
averaged & $\mathrm{R}: 0.67$ & $\mathrm{R}: 0.70$ & $\mathrm{R}: 0.72$ & $\mathrm{R}: 0.68$ & $\mathrm{R}: 0.74$ & $\mathrm{R}: 0.76$ \\
& $\mathrm{~F}: 0.71$ & $\mathrm{~F}: 0.73$ & $\mathrm{~F}: 0.75$ & $\mathrm{~F}: 0.72$ & $\mathrm{~F}: 0.76$ & $\mathrm{~F}: 0.78$ \\
\hline Micro- & $\mathrm{P}: 0.77$ & $\mathrm{P}: 0.78$ & $\mathrm{P}: 0.80$ & $\mathrm{P}: 0.79$ & $\mathrm{P}: 0.82$ & $\mathrm{P}: 0.83$ \\
averaged & $\mathrm{R}: 0.72$ & $\mathrm{R}: 0.75$ & $\mathrm{R}: 0.76$ & $\mathrm{R}: 0.73$ & $\mathrm{R}: 0.77$ & $\mathrm{R}: 0.80$ \\
\hline & $\mathrm{F}: 0.74$ & $\mathrm{~F}: 0.76$ & $\mathrm{~F}: 0.78$ & $\mathrm{~F}: 0.76$ & $\mathrm{~F}: 0.80$ & $\mathrm{~F}: 0.82$ \\
\hline
\end{tabular}

Table V: Results on English dataset with our CNN-based model with novel features.

W1-W6 are explained in the text

\begin{tabular}{|c|c|c|c|c|c|c|}
\hline & W1 & W2 & W3 & W4 & W5 & W6 \\
\hline Age 13-17 & $\begin{array}{l}P: 0.70 \\
R: 0.73 \\
F: 0.72\end{array}$ & $\begin{array}{l}P: 0.73 \\
R: 0.75 \\
F: 0.74\end{array}$ & $\begin{array}{l}P: 0.72 \\
R: 0.74 \\
F: 0.73\end{array}$ & $\begin{array}{l}P: 0.71 \\
R: 0.74 \\
F: 0.73\end{array}$ & $\begin{array}{l}P: 0.75 \\
R: 0.77 \\
F: 0.76\end{array}$ & $\begin{array}{l}P: 0.76 \\
R: 0.79 \\
F: 0.78\end{array}$ \\
\hline Age 18-24 & $\begin{array}{l}P: 0.79 \\
\text { R:0.75 } \\
F: 0.77\end{array}$ & $\begin{array}{l}P: 0.83 \\
R: 0.79 \\
F: 0.81\end{array}$ & $\begin{array}{l}P: 0.81 \\
R: 0.77 \\
F: 0.79\end{array}$ & & & $\begin{array}{l}P: 0.86 \\
R: 0.82 \\
F: 0.84\end{array}$ \\
\hline $\begin{array}{l}\text { Age } 25 \\
\text { plus }\end{array}$ & $\begin{array}{l}P: 0.66 \\
R: 0.69 \\
F: 0.68\end{array}$ & $\begin{array}{l}P: 0.67 \\
R: 0.73 \\
F: 0.70\end{array}$ & $\begin{array}{l}P: 0.68 \\
R: 0.74 \\
F: 0.71\end{array}$ & & $\begin{array}{l}P: 0.70 \\
R: 0.73 \\
F: 0.71\end{array}$ & $\begin{array}{l}P: 0.71 \\
R: 0.75 \\
F: 0.73\end{array}$ \\
\hline $\begin{array}{l}\text { Macro- } \\
\text { averaged }\end{array}$ & $\begin{array}{l}P: 0.72 \\
R: 0.72 \\
F: 0.72\end{array}$ & $\begin{array}{l}P: 0.74 \\
\text { R:0.76 } \\
F: 0.75\end{array}$ & $\begin{array}{l}P: 0.74 \\
R: 0.75 \\
F: 0.74\end{array}$ & & $\begin{array}{l}P: 0.77 \\
\text { R:0.77 } \\
\text { F:0.77 }\end{array}$ & $\begin{array}{l}P: 0.78 \\
R: 0.79 \\
F: 0.78\end{array}$ \\
\hline $\begin{array}{l}\text { Micro- } \\
\text { averaged }\end{array}$ & $\begin{array}{l}P: 0.77 \\
R: 0.73 \\
F: 0.75\end{array}$ & $\begin{array}{l}P: 0.78 \\
R: 0.75 \\
F: 0.77\end{array}$ & $\begin{array}{l}P: 0.78 \\
R: 0.76 \\
F: 0.77\end{array}$ & $\begin{array}{l}P: 0.77 \\
R: 0.75 \\
F: 0.76\end{array}$ & $\begin{array}{l}P: 0.80 \\
R: 0.79 \\
F: 0.79\end{array}$ & $\begin{array}{l}P: 0.81 \\
R: 0.82 \\
F: 0.81\end{array}$ \\
\hline
\end{tabular}

Discussion: As can be seen from Tables II and III, which show baseline experiments with SVM, including the most frequently co-occurring words with hashtags in the user's tweets into the bag-of-words model (column 2) actually degrades the performance of age-prediction. Hashtags are used to index keywords or topics so as to categorize tweets and to allow people to easily follow the topics they are interested in. In this experiment, we attempt to capture the topics a user is interested in by finding hashtag-relevant 
words from other tweets that include the same hashtag. In order to overcome the problem of topic drift, we only use tweets in a window of -10 to +10 days from the tweet containing the hashtag. Based on the hypothesis that a person's age is correlated with the topics he is interested in, we expect to see improvement in the accuracy. However, we notice that by bringing in hashtag-relevant words for all hashtags from all 200 recent tweets introduce too much noise: while false negatives decrease resulting in improved recall, false positives increase resulting in poorer precision. Similar observation is made about including words from the URL titles (column 3). Utilizing linguistic, stylometric, and Twitter-specific features along with 1-to-3-grams from tweet texts improve the precision and recall over the basic BoW model. This confirms the sociolinguistic hypothesis that linguistic and stylometric features serve as indicators of person's age. Finally, exploiting predictive lexica which are pre-trained for age, such as EMNLP2014[27] and WWBP[5] help in improving the accuracy slightly. Despite such precreated lexica having potential to improve the accuracy for age prediction, we observe that because such lexica were trained on Facebook and our data is from Twitter, they fail to achieve higher accuracy as expected (since there is a difference in discourse styles of Facebook and Twitter).

Tables IV and V show results of our experiments based on the use of CNN as our classification model in combination with word embeddings for tweet words/phrases. We find that utilizing word embeddings into our CNN model improves on the baseline of using BoW (compare column 1 of Tables $2 \& 3$ with Tables $4 \& 5$ ) since $\mathrm{CNN}$ learns complex features associating different dimensions of word embedding vectors of a tweet word sequence. In the case of URL titles, replacing them with a sequence of corresponding word embedding vectors and finding features from them using convolutional filters improve the performance of the system (compare column 3 of Tables $2 \& 3$ with Tables $4 \& 5$ ). We also notice that instead of directly using HashtagCooccurringSet words for classification, utilizing three vectors $\min$, max, and avg derived from word embeddings of HashtagCooccurringSet yields improvement in precision and recall both (compare column 2 of Tables $2 \& 3$ with Tables $4 \& 5$ ). Since much less noise is included as opposed to the method of including all words, both false positives and false negatives decrease resulting in improvement in precision and recall. Finally, similar to SVM baseline model, including linguistic, stylometric, Twitter-specific features and using external lexica help to improve the accuracy further.

Drop in accuracy for older age groups is observed for both datasets. This is owing to the fact that for older age groups, we have much less data samples. Overall, using our CNNbased architecture along with novel features improves the micro-F1 score by $12.3 \%$ and $6.6 \%$ for Dutch and English datasets, respectively.

\section{Conclusion}

In this paper, we proposed a novel way to include features derived from hashtags and URLs from tweets for age prediction of Twitter users. We show that using distributed representations incorporated into convolutional neural network improve the accuracy over the baseline bag-of-words model. Augmenting these features with features derived from URLs and hashtags further improves the precision and recall. We examined the effect of adding novel features incrementally and conclude that our model outperforms the baseline by $12.3 \%$ and $6.6 \%$ for Dutch and English datasets, respectively. As a prospective work, and in order to strengthen our proposed methodology, a more in depth comparison with state of art algorithms including L1 regularized logistic regression, random forest and Bayes classifier together with more enhanced feature selection task will be carried out.

\section{ACKNOWLEDGMENT}

We would like to thank the anonymous reviewers for their valuable suggestions because of which the technical quality of the work presented in this paper has improved.

This work is partially supported by EU Marie SkodowskaCurie grant No 645706 and EU grant 770469-Cutler. This paper is based on results of a project that has received funding from the European Unions Horizon 2020 research and innovation program under the Marie Skodowska-Curie grant agreement No 645706. This article reflects only the authors' view and the REA is not responsible for any use that may be made of the information it contains.

\section{REFERENCES}

[1] P. Monachesi and de Leeuw T, "Analyzing elderly behavior in social media through language use," in Proceedings of HCI International 2018. Communications in Computer and Information Science., 2018.

[2] P. Eckert, "Age as a sociolinguistic variable," The handbook of sociolinguistics, pp. 151-167, 1997.

[3] D. Nguyen, D. Trieschnigg, A. S. Doğruöz, R. Gravel, M. Theune, T. Meder, and F. De Jong, "Why gender and age prediction from tweets is hard: Lessons from a crowdsourcing experiment," in Proceedings of COLING 2014, the 25th International Conference on Computational Linguistics: Technical Papers, 2014, pp. 1950-1961.

[4] D. Nguyen, R. Gravel, D. Trieschnigg, and T. Meder, "How old do you think i am? a study of language and age in twitter." in ICWSM, 2013.

[5] H. A. Schwartz, J. C. Eichstaedt, M. L. Kern, L. Dziurzynski, S. M. Ramones, M. Agrawal, A. Shah, M. Kosinski, D. Stillwell, M. E. Seligman et al., "Personality, gender, and age in the language of social media: The open-vocabulary approach," PloS one, vol. 8, no. 9, p. e73791, 2013.

[6] A. A. Morgan-Lopez, A. E. Kim, R. F. Chew, and P. Ruddle, "Predicting age groups of twitter users based on language and metadata features," PloS one, vol. 12, no. 8, p. e0183537, 2017. 
[7] F. Al Zamal, W. Liu, and D. Ruths, "Homophily and latent attribute inference: Inferring latent attributes of twitter users from neighbors." ICWSM, vol. 270, p. 2012, 2012.

[8] S. Volkova, Y. Bachrach, M. Armstrong, and V. Sharma, "Inferring latent user properties from texts published in social media." in AAAI, 2015, pp. 4296-4297.

[9] E. Siswanto and M. L. Khodra, "Predicting latent attributes of twitter user by employing lexical features," in Information Technology and Electrical Engineering (ICITEE), 2013 International Conference on. IEEE, 2013, pp. 176-180.

[10] D. Jurgens, Y. Tsvetkov, and D. Jurafsky, "Writer profiling without the writers text," in International Conference on Social Informatics. Springer, 2017, pp. 537-558.

[11] C. Peersman, W. Daelemans, and L. Van Vaerenbergh, "Predicting age and gender in online social networks," in Proceedings of the 3rd international workshop on Search and mining user-generated contents. ACM, 2011, pp. 37-44.

[12] D. Rao, D. Yarowsky, A. Shreevats, and M. Gupta, "Classifying latent user attributes in twitter," in Proceedings of the 2nd international workshop on Search and mining user-generated contents. ACM, 2010, pp. 37-44.

[13] S. Rosenthal and K. McKeown, "Age prediction in blogs: A study of style, content, and online behavior in pre-and postsocial media generations," in Proceedings of the 49th Annual Meeting of the Association for Computational Linguistics: Human Language Technologies-Volume 1. Association for Computational Linguistics, 2011, pp. 763-772.

[14] J. Tam and C. H. Martell, "Age detection in chat," in Semantic Computing, 2009. ICSC'09. IEEE International Conference on. IEEE, 2009, pp. 33-39.

[15] Y. Kim, "Convolutional neural networks for sentence classification," arXiv preprint arXiv:1408.5882, 2014.

[16] Y. Bengio, R. Ducharme, P. Vincent, and C. Jauvin, "A neural probabilistic language model," Journal of machine learning research, vol. 3, no. Feb, pp. 1137-1155, 2003.

[17] R. Collobert, J. Weston, L. Bottou, M. Karlen, K. Kavukcuoglu, and P. Kuksa, "Natural language processing (almost) from scratch," Journal of Machine Learning Research, vol. 12, no. Aug, pp. 2493-2537, 2011.

[18] T. Mikolov, I. Sutskever, K. Chen, G. S. Corrado, and J. Dean, "Distributed representations of words and phrases and their compositionality," in Advances in neural information processing systems, 2013, pp. 3111-3119.

[19] J. W. Pennebaker and L. D. Stone, "Words of wisdom: Language use over the life span." Journal of personality and social psychology, vol. 85, no. 2, p. 291, 2003.

[20] F. Barbieri, "Patterns of age-based linguistic variation in american english," Journal of sociolinguistics, vol. 12, no. 1, pp. 58-88, 2008.

[21] S. Argamon, M. Koppel, J. W. Pennebaker, and J. Schler, "Mining the blogosphere: Age, gender and the varieties of self-expression," First Monday, vol. 12, no. 9, 2007.
[22] S. Goswami, S. Sarkar, and M. Rustagi, "Stylometric analysis of bloggers age and gender," in Third International AAAI Conference on Weblogs and Social Media, 2009.

[23] J. Schler, M. Koppel, S. Argamon, and J. W. Pennebaker, "Effects of age and gender on blogging." in AAAI spring symposium: Computational approaches to analyzing weblogs, vol. 6, 2006, pp. 199-205.

[24] J. D. Burger and J. C. Henderson, "An exploration of observable features related to blogger age." in AAAI Spring Symposium: Computational Approaches to Analyzing Weblogs. Menlo Park, CA, 2006, pp. 15-20.

[25] U. Pfeil, R. Arjan, and P. Zaphiris, "Age differences in online social networking-a study of user profiles and the social capital divide among teenagers and older users in myspace," Computers in Human Behavior, vol. 25, no. 3, pp. 643-654, 2009.

[26] J. Y. Jang, K. Han, P. C. Shih, and D. Lee, "Generation like: comparative characteristics in instagram," in Proceedings of the 33rd Annual ACM Conference on Human Factors in Computing Systems. ACM, 2015, pp. 4039-4042.

[27] M. Sap, G. Park, J. Eichstaedt, M. Kern, D. Stillwell, M. Kosinski, L. Ungar, and H. A. Schwartz, "Developing age and gender predictive lexica over social media," in Proceedings of the 2014 Conference on Empirical Methods in Natural Language Processing (EMNLP), 2014, pp. 11461151.

[28] Y. Sun, L. Zhu, G. Wang, and F. Zhao, "Multi-input convolutional neural network for flower grading," Journal of Electrical and Computer Engineering, vol. 2017, 2017.

[29] D. P. Kingma and J. Ba, "Adam: A method for stochastic optimization," arXiv preprint arXiv:1412.6980, 2014.

[30] N. Srivastava, G. Hinton, A. Krizhevsky, I. Sutskever, and R. Salakhutdinov, "Dropout: A simple way to prevent neural networks from overfitting," The Journal of Machine Learning Research, vol. 15, no. 1, pp. 1929-1958, 2014. 